\title{
Insights into the inhibitory potential of selective phytochemicals against Mpro of 2019-nCoV: a computer-aided study
}

\author{
Nouman Rasool ${ }^{1,4}$ - Ammara Akhtar ${ }^{2}$. Waqar Hussain ${ }^{3,4}$ \\ Received: 17 March 2020 / Accepted: 13 April 2020 / Published online: 1 May 2020 \\ (C) Springer Science+Business Media, LLC, part of Springer Nature 2020
}

\begin{abstract}
At the end of December 2019, a novel strain of coronavirus, given the name of 2019-nCoV, emerged for exhibiting symptoms of severe acute respiratory syndrome. The virus is spreading rapidly in China and around the globe, affecting thousands of people leading to a pandemic. To control the mortality rate associated with the 2019-nCoV, prompt steps are needed. Until now there is no effective treatment or drug present to control its life-threatening effects in the humans. The scientist is struggling to find new inhibitors of this deadly virus. In this study, to identify the effective inhibitor candidates against the main protease (Mpro) of 2019-nCoV, computational approaches were adopted. Phytochemicals having immense medicinal properties as ligands were docked against the Mpro of 2019-nCoV to study their binding properties. ADMET and DFT analyses were also further carried out to analyze the potential of these phytochemicals as an effective inhibitor against Mpro of 2019-nCoV.
\end{abstract}

Keywords 2019-nCoV $\cdot$ Main protease $\cdot$ Phytochemicals $\cdot$ Docking $\cdot$ ADMET $\cdot$ DFT

\section{Introduction}

nCoronavirus (2019-nCoV) breakout took place in December 2019 in Wuhan city of China. Initially, a lot of cases of unknown etiology regarding pneumonia were reported. Reported patients worked or lived near the local Huanan seafood wholesales market. Associated symptoms are acute respiratory infection and, in some patients, rapidly developing acute respiratory distress syndromes (ARDS), severe acute respiratory syndromes (SARS), Middle East Respiratory Syndrome (MERS), acute respiratory failure, and other serious complication. Mild symptoms are seen in most patients with good prognosis. A lot of casualties are reported of those

Nouman Rasool

noumanrasool@gmail.com

1 Dr Panjwani Center for Molecular Medicine and Drug Research, International Center for Chemical and Biological Sciences, University of Karachi, Karachi 75270, Pakistan

2 Department of Life Sciences, University of Management and Technology, Lahore, Pakistan

3 National Center of Artificial Intelligence, Punjab University College of Information Technology, University of the Punjab, Lahore, Pakistan

4 Center for Professional Studies, Lahore, Pakistan patients having symptoms of severe pneumonia, pulmonary edema, acute respiratory distress syndrome, or multiple organ failure. Chinese Center for Disease Control and Prevention (CDC) identified novel coronavirus on January 7, from throat swab sample of a patient. World Health Organization (WHO) named this virus as 2019-nCoV. Currently, epidemiological and clinical attributes of 2019-nCoV are freighting causing alarming situation globally [1].

Coronavirus is classified into $\alpha$ (including human alphacoronavirus 229E, NL63), $\beta$ (including beta-coronavirus OC43, and HKU1), $\gamma$, and $\delta$ genera. These viruses are identified in a wide range of animal species. In humans, there are six previously reported human coronaviruses that can be transmitted between humans, which are responsible for causing mild upper respiratory disorders. SARS having $10 \%$ fatality rate and MERS with $35 \%$ fatality rate are also beta-categorized coronavirus and are highly pathogenic in humans. Both viruses are of zoonotic origin [2-4]. SARS outbreak took place in 2003, transmitted through mammals into humans especially through bats [5]. Novel coronavirus 2019-nCoV contains Protease Mpro encoded by RNA of the virus, initially reported in 2019 in the city of China, Wuhan. From Wuhan, seafood market pneumonia virus' genome sequence was determined (NCBI genome ID MN908947, GenBank MN908947.3) published by Wu Et al. (LOCUS MN90894, 23-JAN-2020) by multiple sequence alignments with known SARS proteases. It showed 
similarity with SARS crystal structure with the highest accuracy [6]. Mpro is considered a potent target for developing viral inhibitor drugs toward coronavirus [7].

Coronavirus is spreading very quickly; it would be a more reasonable and attractive strategy to develop wide-spectrum inhibitory drugs against this virus, instead of following individual strategy for drug designing. This type of drug development would provide the first line of defense against future emerging CoV-associated ailments like SARS. Development of wide-spectrum drugs requires possible conserved target sequence within whole genus coronavirus. For identifying the possible potent target, extensive research was done and Mpro (molecular weight $34 \mathrm{kDa}$ ) was identified as targeted protease, overall controlling RNA replication and transcription. Mpro is the main CoV protease, sharing highly conservative substrate-recognition pocket by comparing four crystal structures. Homology model also represents all three genetic clusters of genus coronavirus. $\mathrm{CoV}$ genome sequence mutates with high frequency [8]. For all known RNA viruses, coronaviruses have the largest genome ranges from 26 to $32 \mathrm{~kb}$ in length. Apart from encoding structural proteins, the major portion of the viral genome is transcribed and translated into a polypeptide that ultimately encodes for those essential proteins involving in viral reapplication and genome expression. The $\sim 306$ aa length protease is also encoded by polypeptide, and this polypeptide is processed finally into a functional protein. Picornavirus and Mpro shares similar cleavagesite specificity, so this Mpro is also known as 3C-like protease (3CLpro). Extensive research showed that Mpro from different coronaviruses is highly conserved in terms of 3D structure and structure, which is why Mpro is a potential target for designing anti-coronaviral drug [9].

Bioinformatics that is an interdisciplinary field of mathematics, science, and computer science provides very meaningful results regarding the analysis of exome sequencing [10]. Computational tools make sure to assess users from data storage to data retrieval, data analysis, its annotation, and ultimately provide visualization of results for the understanding of biological system thoroughly [11-14]. In silico methods use computational approaches that are cost-effective and are predictive methods for chemical compounds before carrying out a scientific laboratory experiment [15]. Computational tools have a lot of worth today [16-20]. Meaningful outcomes coming from these computational tools give us basic research in the biomedical regime. With time, more and more databases are being included in the study [21]. In this study, computationalbased approaches are utilized to identify the potent inhibiting candidates of Mpro of 2019-nCoV. Through molecular docking and DFT-based computations, reactivity and binding of compounds are analyzed with Mpro of 2019-nCoV, while ADMET properties are computed to represent their suitability for human administration.

\section{Material and methods}

The overall flow of methodology opted in this study is shown in Fig. 1.

\section{Ligands and receptor collection}

A vast range of phytochemicals having alkaloid, flavonoid, terpenoid, and sterol nature was gathered through extensive study of scientific literature. A total of 2750 plant-extracted compounds were collected, with previously reported biological and pharmacological characteristics, inhabited locally in Pakistan and India (sub-continent region). PubChem database was used to retrieve 2D and 3D structures of phytochemicals. For further use, phytochemical files were converted into PDB format by using Discovery Studio [22]. Mpro (PDB ID 6LU7), PDB file of this protein, was downloaded from RCSB Protein Data Bank. For 3D visualization of protease Mpro and its preprocessing (removal of water and ligands), UCSF Chimera software was used [23].

\section{Predicting drug-likeness and ADMET properties}

ADMET (absorption, distribution, metabolism, excretion, and toxicity) properties of selected phytochemicals were studied through PreADMET server for screening to select those lead compounds having drug-like properties against 2019-nCoV. Lipinski's rule of five was used to estimate the drug-likeness properties of selected phytochemicals. Pharmacokinetics and pharmacodynamics characteristics of various phytochemicals are analyzed by evaluating their drug-likeness characteristics. The following set of criteria was used for compound filtering: Lipinski's violation $=0$, solubility $=$ high, GI absorption $=$ high or moderate, blood-brain barrier permeability $=$ no, and toxicity $=$ zero/nil.

\section{Molecular docking and binding energy values evaluation}

Molecular docking of protease Mpro (6LU7) was done against selected 108 phytochemicals that were extracted from various plant species using computational tools like AutoDock and AutoDock Vina. AutoDock tool combines ligand and receptor's interaction information by providing meaningful results. To perform docking, PDBQT files for both phytochemicals and protease Mpro are made through AutoDock tool. This format is required to run Vina command, and finally, binding affinity values of selected phytochemicals against the receptor (protein) is attained, only compounds with the highest binding affinity values are selected for DFT analysis. The grid size of Mpro was $40 \times 44 \times 62 \AA$ A with dimensions $x=-14.152, y=13.429$, and $z=67.842$. 


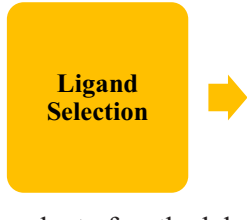

Fig. 1 Flowchart of methodology

\section{DFT analysis and study of reactivity}

To check out the reactivity of selected plant extracted compounds, density-functional theory (DFT)-based computations were performed against receptor protein. It is performed for computation of quantum mechanical calculations of most reactive and active compound [24] DFT by applying Becke, 3parameter, which uses the highest occupied molecular orbital (HOMO) and the lowest unoccupied molecular orbital (LUMO) [25]. B3LYP functional method was applied for DFT calculations, while for calculating band energy gap, $(\Delta \mathrm{E})$ expression of $\mathrm{E}_{\mathrm{LUMO}}-\mathrm{E}_{\mathrm{HOMO}}$ was used [6]. For all DFT-based computations, the ORCA program was used for energy calculations.

\section{Results}

\section{ADMET results}

Phytochemicals were screened to analyze the ADMET properties. ADMET analysis is primarily carried out to identify the potentials of these compounds to be used as a human drug in the future during clinical experiments. The detailed ADMET analysis of the 2750 phytochemicals, in which, a total of 1061 phytochemicals did not follow the Lipinski's rule. The remaining 1689 compounds were further analyzed using the approach BBB (blood-brain barrier) permeability. Out of 1689 compounds, 756 phytochemicals exhibited the properties of non-BBB permeability. In these compounds, a total of 650 compounds showed high GI absorption and solubility characteristics. The toxicity, as well as carcinogenicity properties of the phytochemicals, was studied. After a thorough ADMET analysis, a total of 108 compounds were screened out in the end based on their drug-likeness properties. The docking of 108 phytochemicals was then performed to study the binding properties of ligands and the target protein.

\section{Molecular docking of phytochemicals against Mpro of 2019-nCoV}

After the screening of a vast variety of phytochemicals, 108 phytochemicals were docked against the target protein named Mpro of 2019-nCoV. The binding properties were calculated using AutoDock tool and AutoDock Vina. All compounds showed the binding potential and interact with the Mpro. A cut-off value was applied, and the compounds having binding affinity $\geq-6.5 \mathrm{kcal} / \mathrm{mol}$ values were selected. Out of 108 phytochemicals, 60 compounds were obtained after applying the cut-off value. The compounds named Robustone and SchizolaenoneB having binding affinity value of $-7.8 \mathrm{kcal} /$ mol $(\mathrm{Ki}=1.889 \mu \mathrm{M})$ exhibited strong potential as inhibitors of Mpro activity. This was followed by Osajin and IsosilybinA which exhibited the binding affinity value of $-7.7 \mathrm{kcal} / \mathrm{mol}$ $(\mathrm{Ki}=2.237 \mu \mathrm{M})$. All selected compounds with binding affinity values ranging from -7.8 to $-6.5 \mathrm{kcal} / \mathrm{mol}$ have shown enormous potential to be used as inhibitors against the Mpro of the recent strain of coronavirus (Table 1). Diagrams of the interaction of phytochemicals were studied through discovery studio, as shown in Fig. 2.

\section{DFT (density-functional theory) analysis}

DFT analysis was performed to analyze the reactivity characteristics of the phytochemicals. The complexes were analyzed for reactivity based on the band energy gaps. Among all the analyzed complexes, the ligands showed various LUMO and HOMO energies, thus, the band energy gaps also differed. However, minimum band energy gaps were observed for Robustone $(0.112 \mathrm{kcal} / \mathrm{mol})$, SchizolaenoneB $(0.112 \mathrm{kcal} /$ $\mathrm{mol})$, Osajin $(0.113 \mathrm{kcal} / \mathrm{mol})$, IsosilybinA $(0.114 \mathrm{kcal} / \mathrm{mol})$, SilybinA $(0.115 \mathrm{kcal} / \mathrm{mol})$, Silydianin $(0.117 \mathrm{kcal} / \mathrm{mol})$, and Isopomiferin $(0.118 \mathrm{kcal} / \mathrm{mol})$. Therefore, these results show that the aforementioned showed the highest reactivity with the Mpro of 2019-nCoV. Comprehensive results are shown in Table 2.

\section{Discussion}

Coronaviruses are the pathogenic viruses belonging to coronaviridae family. They are enveloped positive stranded RNA viruses. The name Corona has been given based on spike-like protein protruding out from the viral envelopes, thus making a corona-like shape [26]. They exhibit a wide spectrum of life-threatening symptoms in patients, predominantly affecting the respiratory and gastrointestinal part. There are six different types of the strain of coronavirus which affect the humans causing complications ranging from flu to severe acute respiratory syndrome (SARS) and MERS [27]. 
Table 1 Binding affinity values after applying cut off of $-6.5 \mathrm{kcal} / \mathrm{mol}$ for phytochemicals docked against Mpro of 2019-nCoV

\begin{tabular}{|c|c|c|c|c|c|c|c|}
\hline No. & Compounds & Binding affinity $(\mathrm{kcal} / \mathrm{mol})$ & $\mathrm{Ki}(\mu \mathrm{M})$ & No. & Compounds & Binding affinity $(\mathrm{kcal} / \mathrm{mol})$ & $\mathrm{Ki}(\mu \mathrm{M})$ \\
\hline 1. & Robustone & -7.8 & 1.889 & 31. & TanetinB & -7.0 & 7.299 \\
\hline 2. & SchizolaenoneB & -7.8 & 1.889 & 32. & Sesquiterpene glycoside & -6.9 & 8.643 \\
\hline 3. & Osajin & -7.7 & 2.237 & 33. & Raddeanine & -6.9 & 8.643 \\
\hline 4. & IsosilybinA & -7.7 & 2.237 & 34. & IsoerysenegalenseinE & -6.9 & 8.643 \\
\hline 5. & SilybinA & -7.6 & 2.648 & 35. & Wighteone & -6.9 & 8.643 \\
\hline 6. & Silydianin & -7.6 & 2.648 & 36. & Diprenyleriodictyol & -6.9 & 8.643 \\
\hline 7. & Isopomiferin & -7.6 & 2.648 & 37. & Fumaritine $\mathrm{N}$-oxide & -6.8 & 10.234 \\
\hline 8. & Papraine & -7.5 & 3.136 & 38. & $5,7,4^{\prime}$-Trihydroxyflavone & -6.8 & 10.234 \\
\hline 9. & Paprarine & -7.5 & 3.136 & 39. & Luteolin & -6.8 & 10.234 \\
\hline 10. & SigmoidinC & -7.5 & 3.136 & 40. & ApigeninB & -6.8 & 10.234 \\
\hline 11. & SilybinB & -7.5 & 3.136 & 41. & Tanetin & -6.8 & 10.234 \\
\hline 12. & SilybinC & -7.5 & 3.136 & 42. & AbyssinoneV & -6.8 & 10.234 \\
\hline 13. & IsosilybinB & -7.5 & 3.136 & 43. & Laburnetin & -6.8 & 10.234 \\
\hline 14. & Anthraxin & -7.4 & 3.713 & 44. & AmyrisinB & -6.8 & 10.234 \\
\hline 15. & Mundulinol & -7.4 & 3.713 & 45. & Mearnsetin & -6.8 & 10.234 \\
\hline 16. & SilydianinB & -7.4 & 3.713 & 46. & (+)-syringaresinol & -6.7 & 12.118 \\
\hline 17. & Lycopene & -7.3 & 4.397 & 47. & $5,7,3^{\prime}, 4^{\prime}$-Tetrahydroxyflavone & -6.7 & 12.118 \\
\hline 18. & Erycristagallin & -7.3 & 4.397 & 48. & Tamarixetin & -6.7 & 12.118 \\
\hline 19. & Tomentodiplacone B & -7.3 & 4.397 & 49. & Riboflavin & -6.7 & 12.118 \\
\hline 20. & Derrisin & -7.3 & 4.397 & 50. & Menisdaurin & -6.7 & 12.118 \\
\hline 21. & Isomangostin & -7.3 & 4.397 & 51. & Lupiwighteone & -6.7 & 12.118 \\
\hline 22. & Sigmoidin A & -7.2 & 5.206 & 52. & AmyrisinC & -6.7 & 12.118 \\
\hline 23. & Oxysanguinarine & -7.1 & 6.164 & 52. & AmyrisinA & -6.7 & 12.118 \\
\hline 24. & EuchrenoneB & -7.1 & 6.164 & 54. & (+)-medioresinol & -6.6 & 14.348 \\
\hline 25. & Taxifolin & -7.1 & 6.164 & 55. & Papracinine & -6.6 & 14.348 \\
\hline 26. & SilybinD & -7.1 & 6.164 & 56. & Rhamnetin & -6.6 & 14.348 \\
\hline 27. & Hydroxymunduserone & -7.1 & 6.164 & 57. & EryvarinolsA & -6.6 & 14.348 \\
\hline 28. & Cannflavin & -7.1 & 6.164 & 58. & Erythrinins B & -6.5 & 16.989 \\
\hline 29. & SigmoidinB & -7.0 & 7.299 & 59. & OphiopogonanoneG & -6.5 & 16.989 \\
\hline 30. & EryvarinQ & -7.0 & 7.299 & 60. & DoitunggarcinoneC & -6.5 & 16.989 \\
\hline
\end{tabular}

Recently, a novel strain of coronavirus named 2019-nCoV is causing havoc around the world. There are no vaccines, therapy, or drug that has been approved effective until now against this deadly strain of the virus. To avoid a dire consequence, there is an instant need to cope up the situation by identifying potential inhibitors against 2019-nCoV. This study was preliminary carried out to screen effective inhibitors against the Mpro of 2019-nCoV.

In the recent study, 2750 phytochemicals were collected from various literature sites, articles, and online databases based on their massive medicinal characteristics against a vast range of diseases and health complications. The compounds were screened through ADMET analysis based on their drug-likeness properties. The PreADMET server was used which is an online tool for studying the drug-likeness properties of the different compounds. A total of 108 phytochemicals were eventually obtained which further follow through docking and DFT analysis.
Molecular docking provides a paramount source for analyzing the binding properties of compounds based on the computer-aided analysis. The docking of 108 compounds was performed against the target protein Mpro of 2019-nCoV. The AutoDock tool and AutoDock Vina were used for docking purpose. All the compounds exhibited great binding properties with Mpro. A total of 60 compounds were selected after applying cut-off value of $\geq-6.5 \mathrm{kcal} / \mathrm{mol}$. These compounds reported in this study exhibit great potential as future drugs against Mpro of 2019-nCoV. The compound named, Robustone, is a flavonoid compound which has been previously reported as an antioxidant [28], inhibitor against the protease of dengue virus [29], exhibited great binding affinity properties against Mpro of 2019nCoV. Lycopene has been known to be an effective antioxidant [30] as well as an anti-proliferative 


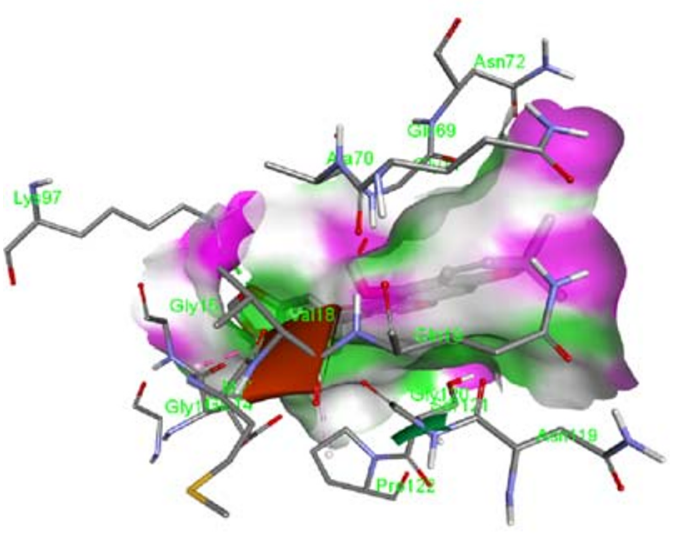

(a)

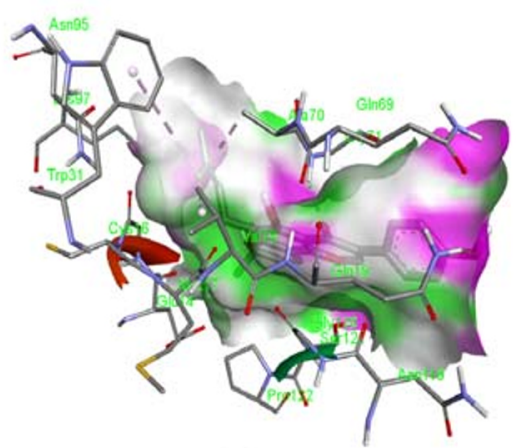

(c)

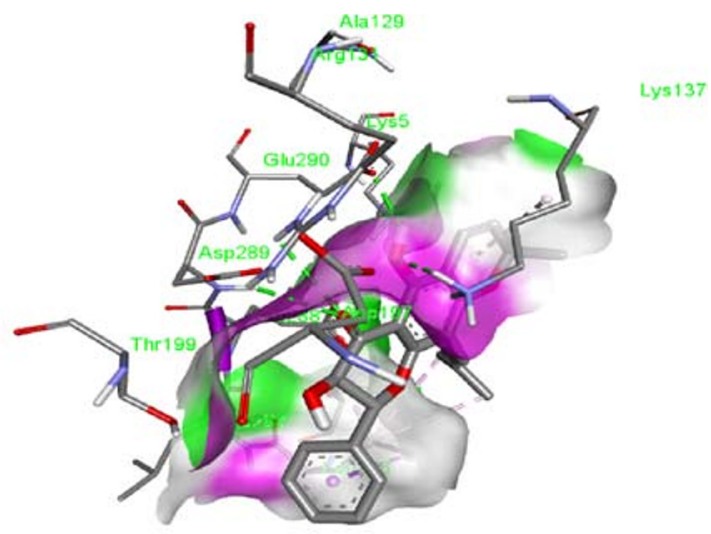

(b)

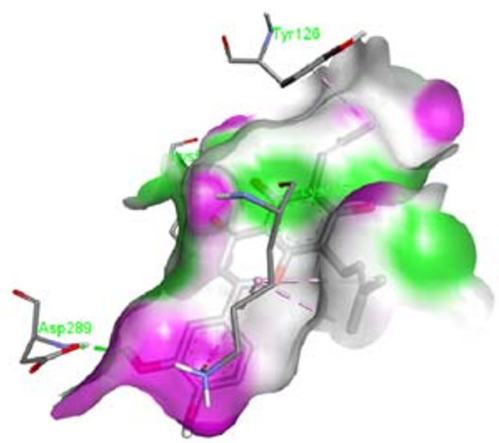

(d)

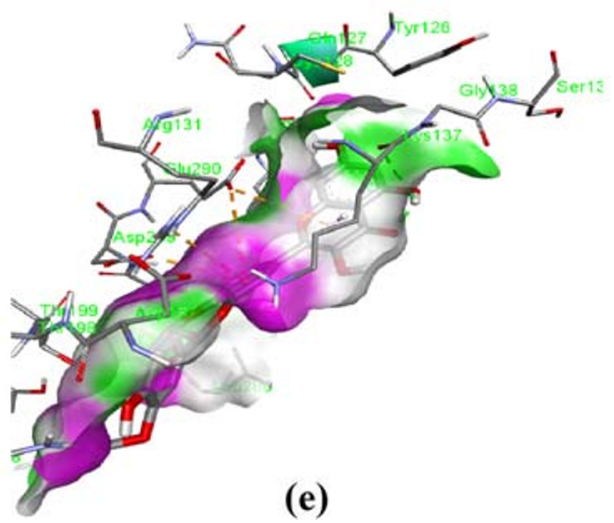

Fig. 2 Interactions of phytochemicals within the binding pocket of Mpro of 2019-nCoV a Robustone, b SchizolaenoneB, c Osajin, d IsosilybinA, and e SilybinA

compound against several cancers [31]. Isopomiferin which is a derivative of plant-based compound pomiferin has been known to exhibit anti-microbial properties, anti-inflammatory agent, effective agent against diabetes and a potent antioxidant (Table 1) [32, 33].

Density functional theory is a computer-based approach with procuring immense popularity in the field of in silico analysis. The analysis of the electronic characteristics of the compounds plays an integral role in understanding their pharmacological properties. In DFT analysis, HOMO and LUMO energies of the compounds were calculated by using computer-based program ORCA. The gap energies exhibiting the molecular reactivity of the compounds were calculated. As the gap energy increases, it leads to compound with decrease reactivity and vice versa [34]. Herein, Robustone, SchizolaenoneB, Osajin, IsosilybinA, SilybinA, Silydianin, and Isopomiferin showed the highest reactivity by depicting minimal band energy gaps. These results were also correlated with the binding affinity and such correlation has already been proved and reported by Hussain et al. [35]. 
Table 2 Results of DFT-based band-energy-gaps computations

\begin{tabular}{|c|c|c|c|c|c|c|c|}
\hline Compounds & $\begin{array}{l}\text { ELUMO } \\
(\mathrm{kcal} / \mathrm{mol})\end{array}$ & $\begin{array}{l}\text { EHOMO } \\
(\mathrm{kcal} / \mathrm{mol})\end{array}$ & $\begin{array}{l}\text { Band energy gap } \\
(\Delta \mathrm{E})(\mathrm{kcal} / \mathrm{mol})\end{array}$ & Compounds & $\begin{array}{l}\text { ELUMO } \\
\text { (kcal/mol) }\end{array}$ & $\begin{array}{l}\text { EHOMO } \\
(\mathrm{kcal} / \mathrm{mol})\end{array}$ & $\begin{array}{l}\text { Band energy gap } \\
(\Delta \mathrm{E})(\mathrm{kcal} / \mathrm{mol})\end{array}$ \\
\hline Robustone & -0.249 & -0.361 & 0.112 & TanetinB & -0.265 & -0.401 & 0.137 \\
\hline SchizolaenoneB & -0.231 & -0.343 & 0.112 & Sesquiterpene glycoside & -0.300 & -0.437 & 0.137 \\
\hline Osajin & -0.290 & -0.402 & 0.113 & Raddeanine & -0.263 & -0.402 & 0.139 \\
\hline IsosilybinA & -0.149 & -0.263 & 0.114 & IsoerysenegalenseinE & -0.277 & -0.416 & 0.139 \\
\hline SilybinA & -0.275 & -0.391 & 0.115 & Wighteone & -0.158 & -0.298 & 0.140 \\
\hline Silydianin & -0.292 & -0.410 & 0.117 & Diprenyleriodictyol & -0.175 & -0.321 & 0.146 \\
\hline Isopomiferin & -0.274 & -0.392 & 0.118 & Fumaritine N-oxide & -0.177 & -0.323 & 0.146 \\
\hline Papraine & -0.268 & -0.387 & 0.119 & $\begin{array}{l}5,7, \\
\text { 4'-Trihydroxyflavone }\end{array}$ & -0.203 & -0.349 & 0.147 \\
\hline Paprarine & -0.280 & -0.399 & 0.119 & Luteolin & -0.163 & -0.310 & 0.147 \\
\hline SigmoidinC & -0.292 & -0.413 & 0.121 & ApigeninB & -0.267 & -0.413 & 0.147 \\
\hline SilybinB & -0.253 & -0.374 & 0.121 & Tanetin & -0.263 & -0.411 & 0.147 \\
\hline SilybinC & -0.158 & -0.280 & 0.122 & AbyssinoneV & -0.222 & -0.370 & 0.148 \\
\hline IsosilybinB & -0.270 & -0.392 & 0.122 & Laburnetin & -0.263 & -0.411 & 0.148 \\
\hline Anthraxin & -0.171 & -0.295 & 0.124 & Amyrisin B & -0.144 & -0.292 & 0.149 \\
\hline Mundulinol & -0.280 & -0.404 & 0.124 & Mearnsetin & -0.140 & -0.289 & 0.149 \\
\hline SilydianinB & -0.298 & -0.423 & 0.126 & (+)-syringaresinol & -0.127 & -0.277 & 0.150 \\
\hline Lycopene & -0.147 & -0.273 & 0.126 & $\begin{array}{l}5,7,3^{\prime}, \\
\text { 4'-Tetrahydroxyflavo- } \\
\text { ne }\end{array}$ & -0.267 & -0.418 & 0.151 \\
\hline Erycristagallin & -0.274 & -0.400 & 0.126 & Tamarixetin & -0.152 & -0.302 & 0.151 \\
\hline TomentodiplaconeB & -0.143 & -0.270 & 0.127 & Riboflavin & -0.132 & -0.284 & 0.152 \\
\hline Derrisin & -0.184 & -0.312 & 0.127 & Menisdaurin & -0.283 & -0.435 & 0.153 \\
\hline Isomangostin & -0.243 & -0.370 & 0.128 & Lupiwighteone & -0.290 & -0.443 & 0.153 \\
\hline SigmoidinA & -0.176 & -0.305 & 0.128 & AmyrisinC & -0.183 & -0.337 & 0.154 \\
\hline Oxysanguinarine & -0.212 & -0.340 & 0.128 & Amyrisin A & -0.232 & -0.387 & 0.155 \\
\hline EuchrenoneB & -0.249 & -0.378 & 0.129 & (+)-medioresinol & -0.162 & -0.318 & 0.156 \\
\hline Taxifolin & -0.169 & -0.299 & 0.129 & Papracinine & -0.120 & -0.278 & 0.158 \\
\hline SilybinD & -0.186 & -0.318 & 0.132 & Rhamnetin & -0.226 & -0.384 & 0.158 \\
\hline Hydroxymunduserone & -0.149 & -0.283 & 0.134 & EryvarinolsA & -0.228 & -0.387 & 0.159 \\
\hline Cannflavin & -0.174 & -0.309 & 0.134 & Erythrinins B & -0.292 & -0.452 & 0.160 \\
\hline SigmoidinB & -0.255 & -0.390 & 0.135 & OphiopogonanoneG & -0.134 & -0.294 & 0.160 \\
\hline EryvarinQ & -0.212 & -0.348 & 0.135 & DoitunggarcinoneC & -0.150 & -0.310 & 0.161 \\
\hline
\end{tabular}

\section{Conclusion}

Coronaviruses are preliminary zoonotic viruses leading to SARS and pneumonia-like health complications in humans. With the growing recent unrestrained cases of the new strain of 2019$\mathrm{nCoV}$, there is an immediate need to find effective drugs against this life-threatening viral strain. This work was carried out to analyze the medicinal properties of the wide range of plantbased compounds against the target protein Mpro of 2019$\mathrm{nCoV}$. ADMET properties were studied to check the compound's efficacy for human usage in the future, all the phytochemicals exhibited good drug-like properties. Docking was performed to analyze the binding interaction of the ligands (phytochemicals) with the target protein, the selected compounds showed magnificent binding interaction with the target protein. DFT analysis of the phytochemicals also showed satisfactory outcomes. Thus, these compounds can be analyzed through the experiment in the future clinical trials of drugs against 2019-nCoV.

\section{Compliance with ethical standards}

Conflict of interest The authors declare no conflict of interest.

\section{References}

1. Chen N, Zhou M, Dong X, Qu J, Gong F, Han Y, Qiu Y, Wang J, Liu Y, Wei Y (2020) Epidemiological and clinical characteristics of 
99 cases of 2019 novel coronavirus pneumonia in Wuhan, China: a descriptive study. Lancet

2. Cui J, Li F, Shi Z-L (2019) Origin and evolution of pathogenic coronaviruses. Nat Rev Microbiol 17(3):181-192

3. Donnelly CA, Malik MR, Elkholy A, Cauchemez S, Van Kerkhove MD (2019) Worldwide reduction in MERS cases and deaths since 2016. Emerg Infect Dis 25(9):1758

4. Poon L, Guan Y, Nicholls J, Yuen K, Peiris J (2004) The aetiology, origins, and diagnosis of severe acute respiratory syndrome. Lancet Infect Dis 4(11):663-671

5. Hu B, Zeng L-P, Yang X-L, Ge X-Y, Zhang W, Li B, Xie J-Z, Shen X-R, Zhang Y-Z, Wang N (2017) Discovery of a rich gene pool of bat SARS-related coronaviruses provides new insights into the origin of SARS coronavirus. PLoS Pathog 13(11)

6. Middleton E, Kandaswami C, Theoharides TC (2000) The effects of plant flavonoids on mammalian cells: implications for inflammation, heart disease, and cancer. Pharmacol Rev 52(4):673-751

7. Dayer MR, Taleb-Gassabi S, Dayer MS (2017) Lopinavir; a potent drug against coronavirus infection: insight from molecular docking study. Arch Clin Infect Dis 12(4)

8. Yang H, Xie W, Xue X, Yang K, Ma J, Liang W, Zhao Q, Zhou Z, Pei D, Ziebuhr J (2005) Design of wide-spectrum inhibitors targeting coronavirus main proteases. PLoS Biol 3(10)

9. Liu X, Wang X-J (2020) Potential inhibitors for 2019-nCoV coronavirus $\mathrm{M}$ protease from clinically approved medicines. bioRxiv

10. Lelieveld SH, Veltman JA, Gilissen C (2016) Novel bioinformatic developments for exome sequencing. Hum Genet 135(6):603-614

11. Rasool N, Ashraf A, Waseem M, Hussain W, Mahmood S (2019) Computational exploration of antiviral activity of phytochemicals against NS2B/NS3 proteases from dengue virus. Turk J Biochem

12. Rasool N, Hussain W (2019) Three major phosphoacceptor sites in HIV-1 capsid protein enhances its structural stability and resistance against inhibitor: explication through molecular dynamics simulation, Molecular Docking and DFT Analysis. Comb Chem High Throughput Screen. https://doi.org/10.2174/ 1386207323666191213142223

13. Rasool N, Hussain W, Mahmood S (2017) Prediction of protein solubility using primary structure compositional features: a machine learning perspective. J Proteomics Bioinform 10(12):324 328

14. Rasool N, Jalal A, Amjad A, Hussain W (2018) Probing the pharmacological parameters, molecular docking and quantum computations of plant derived compounds exhibiting strong inhibitory potential against NS5 from Zika virus. Braz Arch Biol Technol 61(0). https://doi.org/10.1590/1678-4324-2018180004

15. Vogel HG, Maas J, Mayer D (2006) Drug discovery and evaluation: safety and pharmacokinetic assays; with 125 tables. Springer Science \& Business Media

16. Akhtar A, Hussain W, Rasool N (2019) Probing the pharmacological binding properties, and reactivity of selective phytochemicals as potential HIV-1 protease inhibitors. Univ Sci 24(3):441-464

17. Arif N, Subhani A, Hussain W, Rasool N (2019) In silico inhibition of BACE-1 by selective phytochemicals as novel potential inhibitors: molecular docking and DFT studies. Curr Drug Discov Technol. https://doi.org/10.2174/1570163816666190214161825

18. Hussain W, Ali M, Sohail Afzal M, Rasool N (2018) Penta-1,4Diene-3-one oxime derivatives strongly inhibit the replicase domain of tobacco mosaic virus: elucidation through molecular docking and density functional theory mechanistic computations. J Antivirals Antiretrovirals 10(3). https://doi.org/10.4172/19485964.1000177

19. Hussain W, Qaddir I, Mahmood S, Rasool N (2018) In silico targeting of non-structural 4B protein from dengue virus 4 with spiropyrazolopyridone: study of molecular dynamics simulation, ADMET and virtual screening. Virus Dis 1-10

20. Qaddir I, Rasool N, Hussain W, Mahmood S (2017) Computeraided analysis of phytochemicals as potential dengue virus inhibitors based on molecular docking, ADMET and DFT studies. J Vector Borne Dis 54(3):255

21. Giudice TL, Lombardi F, Santorelli FM, Kawarai T, Orlacchio A (2014) Hereditary spastic paraplegia: clinical-genetic characteristics and evolving molecular mechanisms. Exp Neurol 261:518-539

22. Studio D (2009) Version 2.5. Accelrys Inc, San Diego

23. Pettersen EF, Goddard TD, Huang CC, Couch GS, Greenblatt DM, Meng EC, Ferrin TE (2004) UCSF chimera - a visualization system for exploratory research and analysis. J Comput Chem 25(13): $1605-1612$

24. Kavitha E, Sundaraganesan N, Sebastian S (2010) Molecular structure, vibrational spectroscopic and HOMO, LUMO studies of 4 nitroaniline by density functional method

25. Qin P, Zhu H, Edvinsson T, Boschloo G, Hagfeldt A, Sun L (2008) Design of an organic chromophore for p-type dye-sensitized solar cells. J Am Chem Soc 130(27):8570-8571

26. Liang Q, Li B, Zhang H, Hu H (2019) Complete genome sequences of two porcine deltacoronavirus strains from Henan Province, China. Microbiol Resour Announc 8(10):e01517-e01518

27. Dhama K, Kapoor S, Pawaiya R, Chakraborty S, Tiwari R, Verma A (2014) Ross River virus (RRV) infection in horses and humans: a review. Pak J Biol Sci PJBS 17:768-779

28. Mishra A, Tyagi C, Pandey B, Chakraborty O, Kumar A, Jain A (2016) Structural insights into the mode of action of plant flavonoids as anti-oxidants using regression analysis. Proc Natl Acad Sci India Sect B: Biol Sci 86(4):1023-1036

29. Rasool N, Ashraf A, Waseem M, Hussain W, Mahmood S (2018) Computational exploration of antiviral activity of phytochemicals against NS2B/NS3 proteases from dengue virus. Turk J Biochem 44(3):261-277

30. Karahan İ, Ateşşahin A, Yılmaz S, Çeribaşı A, Sakin F (2005) Protective effect of lycopene on gentamicin-induced oxidative stress and nephrotoxicity in rats. Toxicology 215(3):198-204

31. Amir H, Karas M, Giat J, Danilenko M, Levy R, Yermiahu T, Levy J, Sharoni Y (1999) Lycopene and 1, 25-dihydroxyvitamin D3 cooperate in the inhibition of cell cycle progression and induction of differentiation in HL-60 leukemic cells

32. Janoštíková E, Bartošíková L, Nečas J, Juřica J, Florian T, Bartošík T, Klusakova J, Suchý V, Lišková M, Frydrych M (2005) Effects of pomiferin premedication on the antioxidant status of rats with ischemia-reperfused kidney. Acta Vet Brno 74(4):557-564

33. Kupeli E, Orhan I, Toker G, Yesilada E (2006) Anti-inflammatory and antinociceptive potential of Maclura pomifera (Rafin.) Schneider fruit extracts and its major isoflavonoids, scandenone and auriculasin. J Ethnopharmacol 107(2):169-174

34. Zheng Y, Zheng M, Ling X, Liu Y, Xue Y, An L, Gu N, Jin M (2013) Design, synthesis, quantum chemical studies and biological activity evaluation of pyrazole-benzimidazole derivatives as potent Aurora A/B kinase inhibitors. Bioorg Med Chem Lett 23(12): 3523-3530

35. Hussain W, Amir A, Rasool N (2020) Computer-aided study of selective flavonoids against chikungunya virus replication using molecular docking and DFT-based approach. Struct Chem 1-12

Publisher's note Springer Nature remains neutral with regard to jurisdictional claims in published maps and institutional affiliations. 